\title{
Febuxostat versus allopurinol for patients with gout: is it time to overcome concerns regarding cardiovascular safety?
}

\author{
Dimitrios loannis Patoulias, Michael Doumas
}

Second Propedeutic Department of Internal Medicine, Aristotle University of Thessaloniki, General Hospital "Hippokration", Greece

\section{Dear Editor,}

Gout is the most common inflammatory arthritis in the United States, resulting from hyperuricaemia. The latter association represents an established risk factor for cardiovascular disease and coronary artery disease, as shown in a previous meta-analysis of observational studies in a total of 223,448 patients [1]. In addition, patients with gout due to uncontrolled hyperuricaemia experience an increased risk for non-fatal myocardial infarction (MI) [2], while they might also feature increased risk for stroke [3]. Recent real-world data also highlight the association between gout and atrial fibrillation, which inevitably augments the cardiovascular burden [4].

Allopurinol remains the uric acid-lowering treatment option of first choice, while febuxostat is prescribed when allopurinol is contraindicated or not tolerated [5]. Greater serum urate-lowering properties of febuxostat compared to placebo should be taken into account [6]. Unfortunately, medication adherence among gout patients is poor, associated with age and related co-morbidities [7].

Therefore, we sought to determine the comparative efficacy of febuxostat vs. allopurinol across surrogate cardiovascular outcomes of interest, by pooling data from the 2 dedicated cardiovascular outcome trials available so far $[8,9]$. Our motive for this analysis was the U.S. Food and Drug Administration (FDA) warning raised after the publication of the CARES trial [8] regarding the increased risk for cardiovascular and all-cause death with febuxostat compared to allopurinol.

We assessed the following cardiovascular safety outcomes of interest: cardiovascular death, all-cause death, non-fatal MI, non-fatal stroke, fatal MI, fatal stroke, transient ischaemic attack, hospitalization for heart failure, coronary revascularization, cerebrovascular revascularization, and atrial fibrillation.

We assessed only dichotomous variables; therefore, we calculated the risk ratio with $95 \%$ confidence intervals, using an inverse variance-weighted random effects model. Statistical heterogeneity among studies was assessed by using $\left.\right|^{2}$ statistics. All analyses were performed at the 0.05 significance level, while they were undertaken with RevMan 5.3 software.

Our pooled analysis in a total of 12,318 patients with gout showed that subjects treated with febuxostat compared to those prescribed allopurinol did not experience an increased risk for any of the prespecified surrogate cardiovascular outcomes, as summarized in supplementary Table I.

Table I. Summary of prespecified outcomes for the comparison between febuxostat and allopurinol in patients with gout

\begin{tabular}{|lccc|}
\hline Outcome (febuxostat vs. allopurinol) & Risk ratio $(95 \% \mathrm{Cl})$ & Heterogeneity [\%] & $p$-value \\
\hline Cardiovascular death & $1.13(0.82-1.57)$ & 70 & 0.46 \\
\hline All-cause death & $1.01(0.71-1.45)$ & 88 & 0.94 \\
\hline Fatal myocardial infarction & $1.00(0.30-3.27)$ & 65 & 1.00 \\
\hline Fatal stroke & $1.00(0.54-1.84)$ & 0 & 1.00 \\
\hline Non-fatal myocardial infarction & $0.93(0.78-1.12)$ & 0 & 0.46 \\
\hline Non-fatal stroke & $0.96(0.77-1.20)$ & 0 & 0.73 \\
\hline Hospitalization for heart failure & $1.01(0.83-1.23)$ & 15 & 0.91 \\
\hline Transient ischaemic attack & $1.00(0.60-1.50)$ & 4 & 1.00 \\
\hline Coronary revascularization & $0.98(0.77-1.24)$ & 0 & 0.86 \\
\hline Cerebrovascular revascularization & $0.37(0.11-1.26)$ & 0 & 0.11 \\
\hline Atrial fibrillation & $1.00(0.83-1.21)$ & 0 & 0.99 \\
\hline
\end{tabular}

Cl-confidence interval.

\section{Address for correspondence:}

Dimitrios Ioannis Patoulias, Second Propedeutic Department of Internal Medicine, Aristotle University of Thessaloniki, General Hospital "Hippokration", Greece, Phone: +30-6946900777, Fax: +30-2310225083, e-mail: dipatoulias@gmail.com

Submitted: 14.10.2021; Accepted: 17.11.2021 
Notably, a significant proportion of the enrolled study population had significant cardiovascular co-morbidities (one-third of patients enrolled in the FAST trial and $40 \%$ of the patients enrolled in the CARES trial had pre-existing cardiovascular disease). Heterogeneity was low for the vast majority of the assessed outcomes, except for cardiovascular and all-cause death and fatal MI. The risk of bias was low for both studies.

In conclusion, there is no significant difference across surrogate cardiovascular outcomes of interest between febuxostat and allopurinol in patients with gout and cardiovascular co-morbidities. Febuxostat seems to be a safe treatment alternative to allopurinol, despite initial concerns about its cardiovascular safety.

The authors declare no conflict of interest.

\section{References}

1. Clarson LE, Chandratre P, Hider SL, et al. Increased cardiovascular mortality associated with gout: a systematic review and meta-analysis. Eur J Prev Cardiol 2015; 22: 335-343, DOI: 10.1177/2047487313514895.

2. Liu SC, Xia L, Zhang J, et al. Gout and risk of myocardial infarction: a systematic review and meta-analysis of cohort studies. PLoS One 2015; 10: e0134088, DOI: 10.1371/journal. pone. 0134088 .

3. Wiseman SJ, Ralston SH, Wardlaw JM. Cerebrovascular disease in rheumatic diseases: a systematic review and meta-analysis. Stroke 2016; 47: 943-950, DOI: 10.1161/STROKEAHA.115.012052.

4. Singh JA, Cleveland JD. Gout and the risk of incident atrial fibrillation in older adults: a study of US Medicare data. RMD Open 2018; 4: e000712, DOI: 10.1136/rmdopen-2018-000712.

5. Fan B, Zhang P, Li X. Efficacy and safety of febuxostat versus allopurinol in hyperuricemic patients with or without gout: a metaanalysis. Neuro Endocrinol Lett 2020; 41: 195-204.

6. Borghi C, Agabiti-Rosei E, Johnson RJ, et al. Hyperuricaemia and gout in cardiovascular, metabolic and kidney disease. Eur J Intern Med 2020; 80: 1-11, DOI: 10.1016/j.ejim.2020.07.006.

7. Scheepers LEJM, van Onna M, Stehouwer CDA, et al. Medication adherence among patients with gout: a systematic review and meta-analysis. Semin Arthritis Rheum 2018; 47: 689-702, DOI: 10.1016/j.semarthrit.2017.09.007.

8. White WB, Saag KG, Becker MA, et al., CARES investigators. Cardiovascular safety of febuxostat or allopurinol in patients with gout. N Engl J Med 2018; 378: 1200-1210, DOI: 10.1056/ NEJMoa1710895.

9. Mackenzie IS, Ford I, Nuki G, et al., FAST Study Group. Longterm cardiovascular safety of febuxostat compared with allopurinol in patients with gout (FAST): a multicentre, prospective, randomised, open-label, non-inferiority trial. Lancet 2020; 396: 1745-1757, DOI: 10.1016/S0140-6736(20)32234-0. 